\title{
An investigation into the effect of gradients on the manufacturing fidelity of triply periodic minimal surface structures with graded density fabricated by selective laser melting
}

\author{
Lei Yang ${ }^{\mathrm{a}, \mathrm{b}, 1}$, Massimiliano Ferrucci ${ }^{\mathrm{b}, 1}$, Raya Mertens ${ }^{\mathrm{b}, 1}$, Wim Dewulf ${ }^{\mathrm{b}, 1}$, Chunze Yan ${ }^{\mathrm{a}, *}$, Yusheng Shi ${ }^{\mathrm{a}}$, \\ Shoufeng Yang $\mathrm{b}, \mathrm{c}, 1, * *$ \\ a State Key Laboratory of Materials Processing and Die \& Mould Technology, School of Materials Science and Engineering, Huazhong University of Science and Technology, Wuhan 430074, China \\ ${ }^{\mathrm{b}}$ Department of Mechanical Engineering, KU Leuven (Catholic University of Leuven), 3001 Leuven, Belgium \\ ${ }^{c}$ Materials Research Group, Faculty of Engineering and the Environment, University of Southampton, Southampton, SO17 1BJ, UK
}

\section{A R T I C L E I N F O}

Associate Editor: A. Clare

\section{Keywords}

Graded cellular structure

Selective laser melting

Triply periodic minimal surface

Gyroid

X-ray computed tomography

\begin{abstract}
A B S T R A C T
Triply periodic minimal surfaces (TPMSs) have attracted increasing attention for their high manufacturability, biocompatibility, and mechanical properties. In this work, graded Gyroid cellular structures (GCSs) with varying gradient directions were mathematically designed and manufactured via selective laser melting (SLM). The effect of gradients on manufacturing fidelity, i.e. degree of conformity between the manufactured part and engineering design, of these structures was investigated using X-ray computed tomography (CT). The results indicate that relative density and volume fraction of as-built GCSs are higher than those specified in the engineering design due to strut diameters being larger than specification and the presence of bonded powder particles. Manufacturing fidelity is shown to depend on the geometry of the struts in these structures, including inclination angle of struts, relative density, and density gradient direction. Bonded powder particles were particularly present at the upper inner walls of sphere-like pores, where inclination angles are low and as a result there is a lack of support and non-ideal transfer of heat from the melt pool. Decreasing density along the building direction reduces the occurrence of bonded powder particles and increases manufacturing fidelity. The empirical findings in this study provide insight into the effects of part geometry on the quality of its manufacture by SLM. Results are used to establish guidelines for optimal design of cellular structures.
\end{abstract}

\section{Introduction}

The Gyroid surface, first discovered by Luzzati and Spegt (1967), is one type of triply periodic minimal surfaces (TPMSs) with mean curvature radius of zero, as reported by Scherer (2013). Kapfer et al. (2011) stated that the TPMS cellular structure is considered as the most promising porous/lattice structure for biological applications, due to its structural similarity to bone tissue (e.g. larger surface area, high permeability, interconnected open cell structure); Yan et al. (2012) also experimentally illustrated the high manufacturability of TPMS cellular structures in the additive manufacturing (AM) process.

In recent years, there has been significant interest in TPMS cellular structures, especially Gyroid cellular structures (GCSs). Hao et al. (2011) successfully fabricated GCSs with unit cell sizes ranging from $2 \mathrm{~mm}$ to $8 \mathrm{~mm}$ using selective laser melting (SLM), and demonstrated the high manufacturability of GCSs. Yánez et al. (2018) designed deformed Gyroid structures with non-spherical pores through changing the coefficients in the mathematical equation of Gyroid structures and observed that variant Gyroids possess high stiffness and strength compared with normal Gyroid structures owning spherical pores. Yang et al. (2018) numerically studied GCSs under compressive loading and demonstrated significantly more uniformly distributed stress and strain compared to other lattice structures. Montazerian et al. (2017) performed compression testing of GCSs and suggest that GCSs have great potential for the design and manufacture of graded porosity scaffolds with high permeability and good mechanical properties. Maskery et al. (2018) proposed a novel design method enabling the customization of the graded lattice with relative density gradually changing. Normally, lattice structures consist of only one type of unit cell and their properties are defined by this unit cell. However, hybrid lattices with two or more types of unit cells together are shown in Li et al. (2016)

\footnotetext{
* Corresponding author.

** Corresponding author at: Department of Mechanical Engineering, KU Leuven (Catholic University of Leuven), 3001 Leuven, Belgium.

Email addresses: c_yan@hust.edu.cn (C. Yan); shoufeng.yang@kuleuven.be (S. Yang)

1 Member of Flanders Make.
} 
to efficiently combine properties from the constituent cell types. The method proposed by Maskery also enables hybridising two or more TPMSs unit cells together with a uniform transition in the adjacent regions.

However, Hussein et al. (2013) stated that the SLM-made GCSs exhibit geometrical deviations from engineering design, e.g. an STL file. Al-Saedi et al. (2018) observed that the dimensions of solid struts in manufactured lattice structures were slightly larger than the intended design. Concli and Gilioli (2019) also pointed out that the actual geometry of AM-manufactured structures differs significantly from the design. To the best of our knowledge, literature investigating geometrical deviations in AM porous structures is limited to those deviations resulting from limitations inherent to the AM process. For example, the stair-step effect and the occurrence of bonded powders are typically key factors in defining the manufacturing fidelity of SLM-made parts. The stair-step effect is caused by the finite size of the build layers, as reported by Strano et al. (2013), while the partially molten powders are bonded on the surface due to non-optimal heat transfer from the melt pool, which is illustrated by Wang et al. (2013). Furthermore, Sing et al. (2018) illustrated that the width of molten pool is larger than the laser beam spot size, making the experimental strut dimensions larger than design. Furthermore, Sing et al. (2018) investigated the effects of processing parameters on the dimensional accuracy of cellular lattice structures fabricated by SLM and found that strut dimensions were most sensitive to laser power. Moreover, Mancisidor et al. (2016) pointed out when printing the edges of a part, the scanning speed of the laser is typically slower than the scanning speed when printing internal areas. This phenomenon is due to the laser initiating its straight path from the part boundary, in which it exhibits an initial acceleration phase. The slower speed near the boundaries, therefore, results in higher deposited energy density, which generates melt pools that extend past the intended edge. In consequence, the printed features will have enlarged dimensions.

All the previously-mentioned factors are intrinsic to the AM process. However, the geometry of the printed parts also affects manufacturing fidelity. Kruth et al. (2005) fabricated different shapes commonly used in engineering design by SLM, such as thin wall, hole, cylinder, overhang, sharp corner. The tested accuracy of different shapes was found to differ, with respect to the same process parameters. Sarker et al. (2018) experimentally revealed that the inclination angles of inclined Ti-6Al-4V parts greatly affected the surface topography, morphology, and roughness. The effects of part geometry on the manufacturing fidelity of porous/lattice structures has not been fully investigated, and is therefore the topic of this work. Uniform GCS and continuously graded GCSs were designed and fabricated by SLM. The manufactured parts are measured by X-ray computed tomography (CT) and the tomographic volumes are compared to those of the engineering design. Mathematical equations between geometrical parameters and the presence of overhangs were developed to determine the occurrence of bonded powder particles. Especially, the effect of gradients on manufacturing fidelity in GCSs was discussed. The findings provide a quantitative interpretation of how part geometry affects manufacturing fidelity and are used to optimize the manufacturing of cellular structures.

\section{Experimental methodology}

\subsection{Design process}

Gyroid surfaces can be parameterized using the following mathematical equation.

$$
\begin{aligned}
& \sin \left(\frac{2 \pi}{a} \cdot x\right) \cos \left(\frac{2 \pi}{a} \cdot y\right)+\sin \left(\frac{2 \pi}{a} \cdot y\right) \cos \left(\frac{2 \pi}{a} \cdot z\right) \\
& \quad+\sin \left(\frac{2 \pi}{a} \cdot z\right) \cos \left(\frac{2 \pi}{a} \cdot x\right)=t
\end{aligned}
$$

where $a$ is the unit cell size and parameter $t$ defines relative density $(\mathrm{RD})$, i.e. the ratio of the porous structure density to the density of the matrix material. MATLAB (Mathworks Inc., USA) software was utilized to design the Gyroid unit cell. In this study, three samples of each of the following GCSs are manufactured: uniform GCSs (U-GCS), graded GCSs with RD gradient direction parallel to build direction (G1-GCS), and graded GCSs with RD gradient direction perpendicular to build direction (G2-GCS). All structures are made with unit cell size of $a=4 \mathrm{~mm}$; thus each Gyroid unit cell occupies a volume of $4 \mathrm{~mm} \times 4 \mathrm{~mm} \times 4 \mathrm{~mm}$. Uniform Gyroid unit cells are defined by a constant $\mathrm{RD}$, which is parameterized by a constant $t$ as shown in Fig. 1(a). Graded Gyroid unit cells are defined by a non-constant volume fraction, which is parameterized by a linearly changing $t$ along the gradient direction, as shown in Fig. 1(b). Each manufactured sample comprises five unit cells along each Cartesian direction. The samples are therefore cubic and occupy a total nominal volume of $20 \mathrm{~mm} \times 20 \mathrm{~mm} \times 20 \mathrm{~mm}$. The RD of U-GCS samples is $15 \%$, as shown in Fig. 1(c). The RD in G1-GCS samples decreases linearly from $20 \%$ at the bottom layer to $10 \%$ at the top layer, as shown in Fig. 1(d), while in the G2-GCS samples the RD decreases linearly from $20 \%$ to $10 \%$ along Y, as shown in Fig. 1(e). These structures were also adopted by Yang et al. (2019) to analysis the mechanical properties of graded Gyroid cellular structure in another relevant work.

\subsection{Manufacturing process}

Magics software (Materialise, Leuven, Belgium) was used for file preparation. Samples were manufactured on an M-lab cusing instrument (Concept Laser $\mathrm{GmbH}$, Germany) with a build envelope of $90 \times 90 \times 80$ $\mathrm{mm}^{3}(\mathrm{x}, \mathrm{y}, \mathrm{z}) . \mathrm{A} 100 \mathrm{~W}$ fibre laser was equipped and the spot diameter of this laser beam is $50 \mu \mathrm{m}$. The building chamber was flushed with high purity argon during the scanning process to an oxygen level of lower than $0.3 \%$. The powder used is stainless steel 316L (Praxair Surface Technologies, Inc.) with Gaussian particle size distribution and the volume-based D-values: D10 $=19 \mu \mathrm{m}, \mathrm{D} 50=31 \mu \mathrm{m}$ and D90 $=49 \mu \mathrm{m}$. Based on an initial parameter optimization, the optimized processing parameters for the $316 \mathrm{~L}$ powder are: laser power of $90 \mathrm{~W}$, hatch spacing of $0.077 \mathrm{~mm}$, scanning speed of $600 \mathrm{~mm} / \mathrm{s}$, and layer thickness of $30 \mu \mathrm{m}$. Manufactured samples were removed from the base plate by wire electrical discharge machining (Wire-EDM) Trapped loose powders were removed by ultrasonic cleaning in a bath of pure ethyl alcohol. Fig. 2(a) shows as-built samples before Wire-EDM, while Fig. 2(b) and (c) present the graded Gyroid cellular structures after cutting and cleaning from the substrate. Red arrows in Fig. 2(b) and (c) denote the gradient direction with density gradually decreasing.

\subsection{Measurement and characterization}

The dimensions and weight of as-built samples were measured with a digital Vernier calliper (smallest scale division of $0.01 \mathrm{~mm}$ ) and electronic balance (smallest scale increments of $0.1 \mu \mathrm{g}$ ), respectively. Each dimension was measured three times, each time at different locations on the sample. The average of three positions was used as the dimension value. The RD of the fabricated cellular structure was determined by dividing the measured density of cellular structures by the density of the matrix material $\left(8.00 \mathrm{~g} / \mathrm{cm}^{3}\right.$ for $316 \mathrm{~L}$ stainless steel). Archimedes' principle was applied to calculate the density of solid struts of Gyroid cellular structures from the weight of the fabricated Gyroid cellular structure in air and ethanol. RD of solid struts was calculated by dividing the calculated density of lattice struts by the theoretical density of the parent material $316 \mathrm{~L}$.

For each type of GCS, one sample was CT scanned with a Nikon XT H 225 ST (Nikon Metrology NV, Belgium) to determine the actual volume. CT measurements were performed with $200 \mathrm{kV}$ X-ray source acceleration voltage and $160 \mu \mathrm{A}$ filament current. Detector exposure was set to $1000 \mathrm{~ms}$ and a $0.25 \mathrm{~mm}$ thick Sn filter was used to remove low-energy X-rays, thereby reducing the effects of beam hardening. Two-di- 
(a)

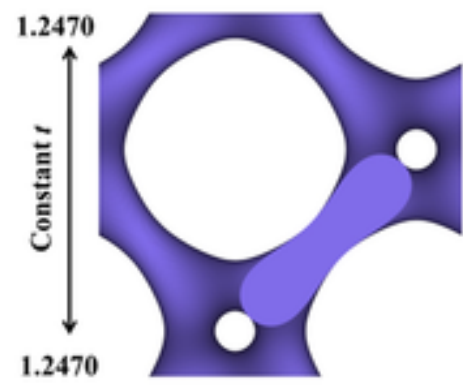

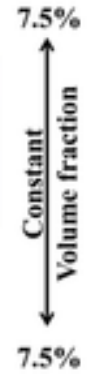

(b)

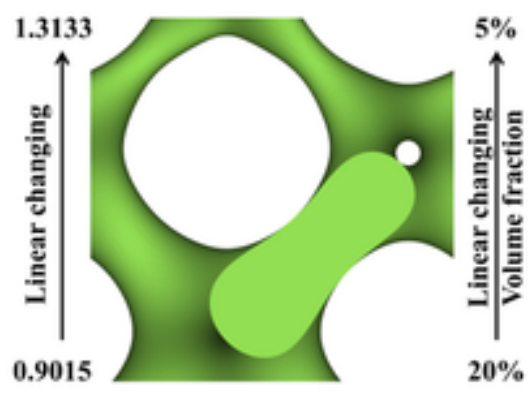

(c)

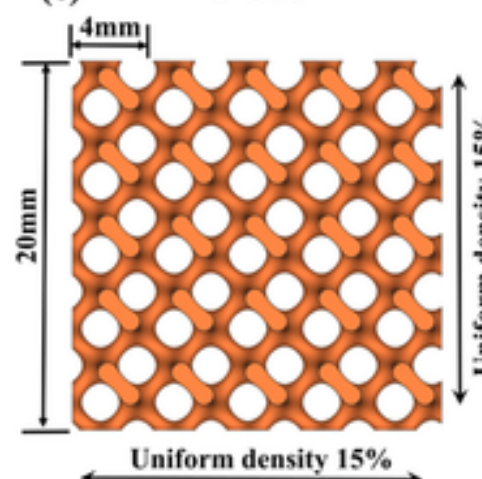

(d)

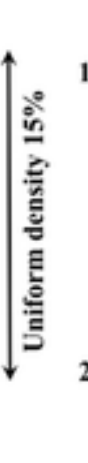

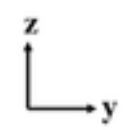

G1-GCS (e)

G2-GCS

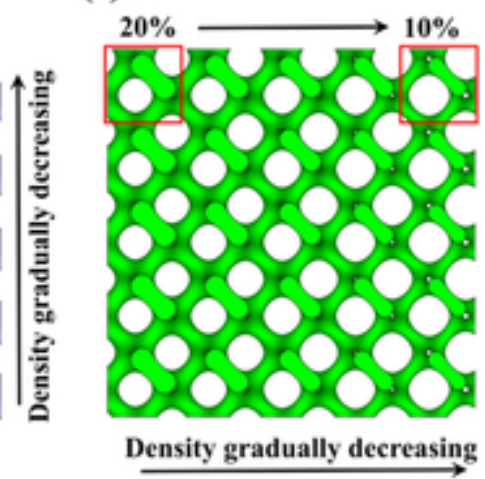

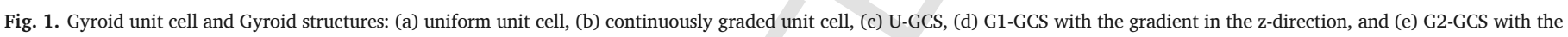
gradient in the $y$-direction.
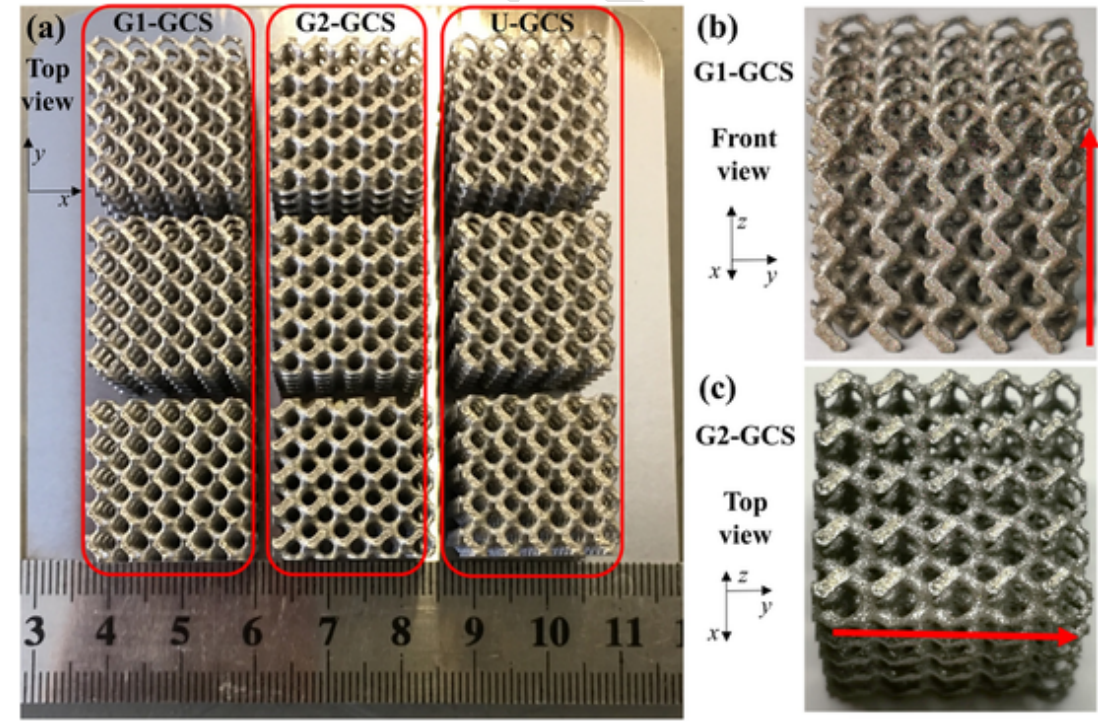

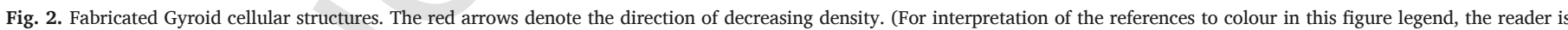
referred to the web version of this article).

mensional (2D) radiographic projections of the cellular structures were acquired at 3142 equally-spaced rotation positions of the sample stage, corresponding to angular increments between adjacent projections of $0.1145^{\circ}$. The structures were tomographically reconstructed with CT Pro 3D (Nikon Metrology NV, Belgium) from the set of acquired radiographic images. The reconstructed volumetric model consists of isotropic voxels with side lengths of $19.5 \mu \mathrm{m}$; each voxel is assigned a grey value corresponding to the X-ray attenuation of the material(s) contained within the voxel. Further analysis and visualization were performed on commercially available volumetric analysis software VG Stu- dio MAX 3.1 (Volume Graphics GmbH, Germany). The surface of the structure is determined by defining a grey value threshold. The volume of the structure can then be calculated from the resulting surface model. The volume fraction (VF), which is equivalent to RD for homogeneous material, was determined by dividing the previously measured volume by the total cubic volume of the sample. RD of solid struts was assessed by conducting the porosity analysis in VG Studio Max 3.1 using the custom method VGEasyPore with automatic settings (the threshold for the minimal size of voids is 3 voxels). 


\section{Results and discussion}

\subsection{Relative density and volume fraction of as-built GCSs}

Table 1 shows the measured dimensions, weight, and calculated relative densities of fabricated GCS samples. The results indicate that SLM manufacturing of GCSs is repeatable. Relative densities of struts tested by Archimedes' principle show that high relative densities (above 98.5\%) of the struts can be achieved for the 316 L GCSs, while the porosity analysis on the CT-reconstructed models show that nearly no pore was found in struts under the current scanning accuracy (isotropic voxel side length $=19.5 \mu \mathrm{m}$ ), as shown in Fig. 3 .

The measured dimensions of the manufactured GCSs deviate from their engineering designs: dimensions along $\mathrm{X}$ and $\mathrm{Y}$ are consistently larger than their designed specifications, while the measured dimensions along $\mathrm{Z}$ are consistently smaller, likely due to the loss of height when the components are removed from the baseplate. For each GCS, sample number 2 was measured by CT. The CT-measured material volumes are listed in Table 2; VF is determined by dividing the CT-measured volumes by the corresponding total cubic volumes from Table 1 .

For both $\mathrm{RD}$ and $\mathrm{VF}$, the percentage deviation is defined as the ratio of the deviation to the engineering design. The deviation of $\mathrm{RD}$ can be calculated by

$\eta_{R D}=\frac{\overline{\rho^{*}}-\rho_{D}^{*}}{\rho_{D}^{*}} \times 100 \%$

in which, $\bar{\rho}^{*}$ is the mean RD of the tested samples, $\rho_{D}^{*}$ is the specified relative density of engineering design. For U-GCS and G2-GCS, the value of $\rho_{D}^{*}$ is $15 \%$, while for G1-GCS the value of $\rho_{\mathrm{D}}^{*} \rho_{D}^{*}$ was modified to $14.92 \%$ to account for the material removal as a result of the EDM process. The deviation of $\mathrm{VF}$ is defined as

$\eta_{V F}=\frac{V-V_{D}}{V_{D}} \times 100 \%$

where, $V$ and $\boldsymbol{V}_{\boldsymbol{D}}$ are the CT-measured volume and engineering designed volume, respectively. The amount of removed material by EDM process should also be considered when specifying engineering designed volume, thus the value differs sample by sample.

The actual RD and VF values in Tables 1 and 2 indicate that both the RD and VF values of as-built samples were higher than the specified value of $15 \%$ due to enlarged strut diameter and adhered powder on the surfaces. The enlarged strut diameter and adhered powder can be attributed to the larger molten pool caused by the lack of cooling, as reported by Hussein et al. (2013). Furthermore, Yang et al. (2018) pointed out that as the specific surface area per unit volume (the ratio of surface area to volume) of the cellular structure is higher than the same ratio for bulk material, the effects of enlarged strut diameter and adhered powder on the surfaces are amplified, resulting in the $\mathrm{RD}$ deviation.

Besides, it is also noticed that the gained $\mathrm{RD}$ and VF values of G1-GCS samples were lower than those of the other two structures. The trend in actual volume and VFs in Table 2 shows consistency with the trend of measured $\mathrm{RD}$ values in Table 1 . The percentage deviations of G2-GCS are similar to those of U-GCS, while the deviations of G1-GCS samples were the lowest. This observation indicates that a gradient along the building direction enhances the manufacturing fidelity, while a gradient perpendicular to the building direction results in similar manufacturing fidelity to the uniform structures.

\subsection{Surface morphologies of the as-built GCSs}

To investigate the deviation distribution of the SLM-made samples, the designed STL model was imported into VG studio and overlapped with the reconstructed model. A typical contour comparison between nominal and actual GCS models on different sections is presented in Fig. 4. It is observed that for the diagonal section in Fig. 4(a) and the vertical section in Fig. 4(b), the bottom contours of fabricated struts exceed the designed profile, resulting in larger strut diameters.

Furthermore, three-dimensional deviation maps between the CT data and engineering designs are generated (shown in Fig. 5(a)). The deviation maps do not indicate obvious defects or broken cells in the as-built GCS. Deviations over most areas of the entire sample area are lower than $0.25 \mathrm{~mm}$. The largest deviations occur at the upper inner walls of sphere-like pores, which is consistent with the contour comparison in Fig. 4. The deviations can be attributed to overhangs, which is a result of lacking support and the lower heat conduction rate of metal powder when compared to the conduction rate of solid metal, as illustrated by Kruth et al. (2007). When the laser irradiates the powder-supported zone, the absorbed energy cannot be fully conducted to the baseplate, resulting in a larger molten pool and adhering more partially melted powders below the working layer. The outline of fabricated overhangs typically exceed the designed profile in a downward direction (with respect to the building direction) and are generally characterized by significantly rougher surfaces. This phenomenon is the same with the previous study reported by Mazur et al., 2016a, where the surface roughness of cooling channels was higher for upper (down-facing) channel surfaces than for lower surfaces due to lack of support. Thus, the geometry of cellular structure affects the manufacturing fidelity, particularly the occurrence of bonded powder particles due to overhangs.

Two-dimensional cross-sections of nominal-actual comparisons between the CT-measured data and the engineering design are plotted in Fig. 5(b). The thin lines perpendicular to the surfaces of isolated struts denote the deviation; the length and color of these lines denotes the magnitude of the deviation. The lines of G1-GCS are shorter than the

Table 1

Dimensions, weight and densities of fabricated GCS samples.

\begin{tabular}{|c|c|c|c|c|c|c|c|}
\hline Designed cellular & $\begin{array}{l}\text { Sample } \\
\text { number }\end{array}$ & $\begin{array}{l}\text { Actual dimensions } \\
\text { of cellular cubic }\end{array}$ & Weight & $\begin{array}{l}\text { RD of } \\
\text { solid } \\
\text { struts }\end{array}$ & $\begin{array}{l}\text { Actual } \\
\mathrm{RD} \\
\rho^{*}\end{array}$ & $\begin{array}{l}\text { Mean } \\
\text { RD } \\
\bar{\rho}^{*}\end{array}$ & $\begin{array}{l}\text { Deviation } \\
\eta_{R D}\end{array}$ \\
\hline & & $\mathrm{X} \times \mathrm{Y} \times \mathrm{Z}(\mathrm{mm})$ & (g) & (\%) & (\%) & $(\%)$ & $(\%)$ \\
\hline \multirow[t]{3}{*}{ U-GCS } & 1 & $20.04 \times 20.02 \times 19.63$ & 10.30 & 98.74 & 16.35 & 16.33 & 8.87 \\
\hline & 2 & $20.04 \times 20.04 \times 19.67$ & 10.32 & 98.77 & 16.33 & & \\
\hline & 3 & $20.04 \times 20.06 \times 19.55$ & 10.25 & 98.70 & 16.30 & & \\
\hline \multirow[t]{3}{*}{ G1-GCS } & 1 & $20.04 \times 20.06 \times 19.61$ & 10.03 & 98.85 & 15.91 & 15.91 & 6.60 \\
\hline & 2 & $20.02 \times 20.04 \times 19.54$ & 9.95 & 98.74 & 15.89 & & \\
\hline & 3 & $20.04 \times 20.04 \times 19.59$ & 10.02 & 98.71 & 15.93 & & \\
\hline \multirow[t]{3}{*}{ G2-GCS } & 1 & $20.06 \times 20.06 \times 19.52$ & 10.25 & 98.80 & 16.32 & 16.34 & 8.93 \\
\hline & 2 & $20.04 \times 20.00 \times 19.61$ & 10.30 & 98.75 & 16.37 & & \\
\hline & 3 & $20.02 \times 20.06 \times 19.61$ & 10.29 & 98.66 & 16.33 & & \\
\hline
\end{tabular}




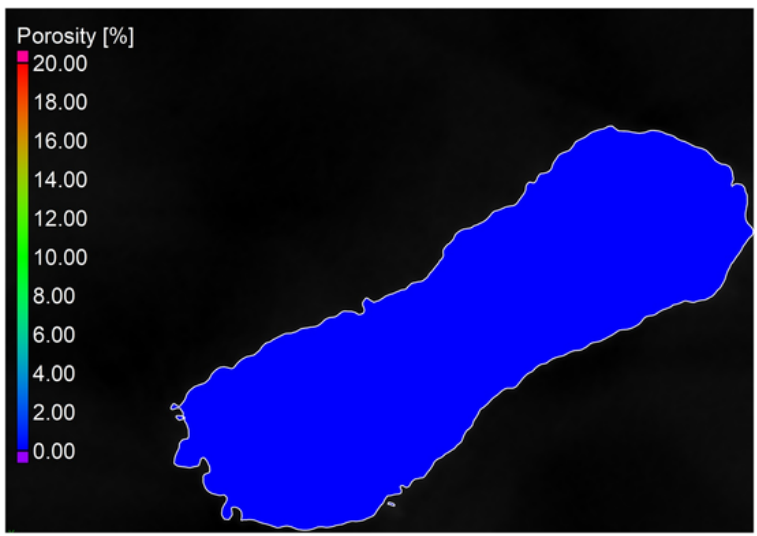

Fig. 3. Porosity analysis of a typical strut on the CT-reconstruction model.

Table 2

Volume and VF of CT reconstructed models.

\begin{tabular}{llll}
\hline & $\begin{array}{l}\text { CT-Volume } \\
\left(\mathrm{mm}^{3}\right)\end{array}$ & $\begin{array}{l}\text { Measured VF } \\
(\%)\end{array}$ & $\begin{array}{l}\text { Deviation } \\
\eta_{\text {VF }}(\%)\end{array}$ \\
\hline U-GCS & 1311.31 & 16.60 & 10.67 \\
G1-GCS & 1249.55 & 15.94 & 7.45 \\
G2-GCS & 1308.07 & 16.64 & 10.93 \\
\hline
\end{tabular}

lines in the other two structures, which indicates that the as-built G1-GCS sample has the highest manufacturing fidelity.

Furthermore, the statistical surface deviations of the uniform and graded GCSs are plotted in Fig. 6. While the surface deviations of GCSs in Fig. 6 appear to be normally distributed, the peak deviation and D50 value of cumulative percentage are not identical and the D10 and
D90 values are not symmetrical with respect to D50 value. The analysis illustrates that the distribution of surface deviations is skewed.

The cumulative percentage lines show that most deviations of GCSs are positive, making the total volume larger than the designed value as shown in Table 2. The peak deviation of U-GCS is $0.028 \mathrm{~mm}$ and the D10, D50, and D90 of cumulative percentage are $-0.024 \mathrm{~mm}, 0.030 \mathrm{~mm}$, and $0.098 \mathrm{~mm}$, respectively. The peak deviation of G2-GCS is $0.021 \mathrm{~mm}$, which is lower than the peak deviation of U-GCS. The D10, D50, and D90 values of G2-GCS are $-0.022 \mathrm{~mm}, 0.022 \mathrm{~mm}$, and $0.099 \mathrm{~mm}$, respectively and are similar to the corresponding values of U-GCS. The peak deviation of G1-GCS is $0.021 \mathrm{~mm}$ and the D10, D50, D90 values are $-0.032 \mathrm{~mm}, 0.230 \mathrm{~mm}$, and $0.091 \mathrm{~mm}$, respectively. Overall, G1-GCS samples have lower manufacturing deviations when compared to U-GCS.

\subsection{Effects of geometry of the struts on manufacturing fidelity}

To further study the influence of geometry of the struts on manufacturing fidelity, a schematic of the cross-section with several powder layers of an inclined strut is shown in Fig. 7(a). The length $l$ of the overhanging area in a cross-section of an inclined strut is given by

$l=\frac{\delta}{\tan \theta}$

where $\delta$ is the powder layer thickness defined by the SLM process and $\theta$ is the inclination angle of the struts, ranging from 0 to 90 degrees.

The extent of overhanging can be described by the overhanging rate,

$$
\eta_{\text {overhanging }}=\frac{l}{D}=\frac{\delta}{D \cdot \tan \theta}
$$

where $D$ is the strut diameter.

Eqs. (4) and (5) indicate that, as the inclination angle decreases, the length of the overhanging area $l$, as well as the extent of overhang-
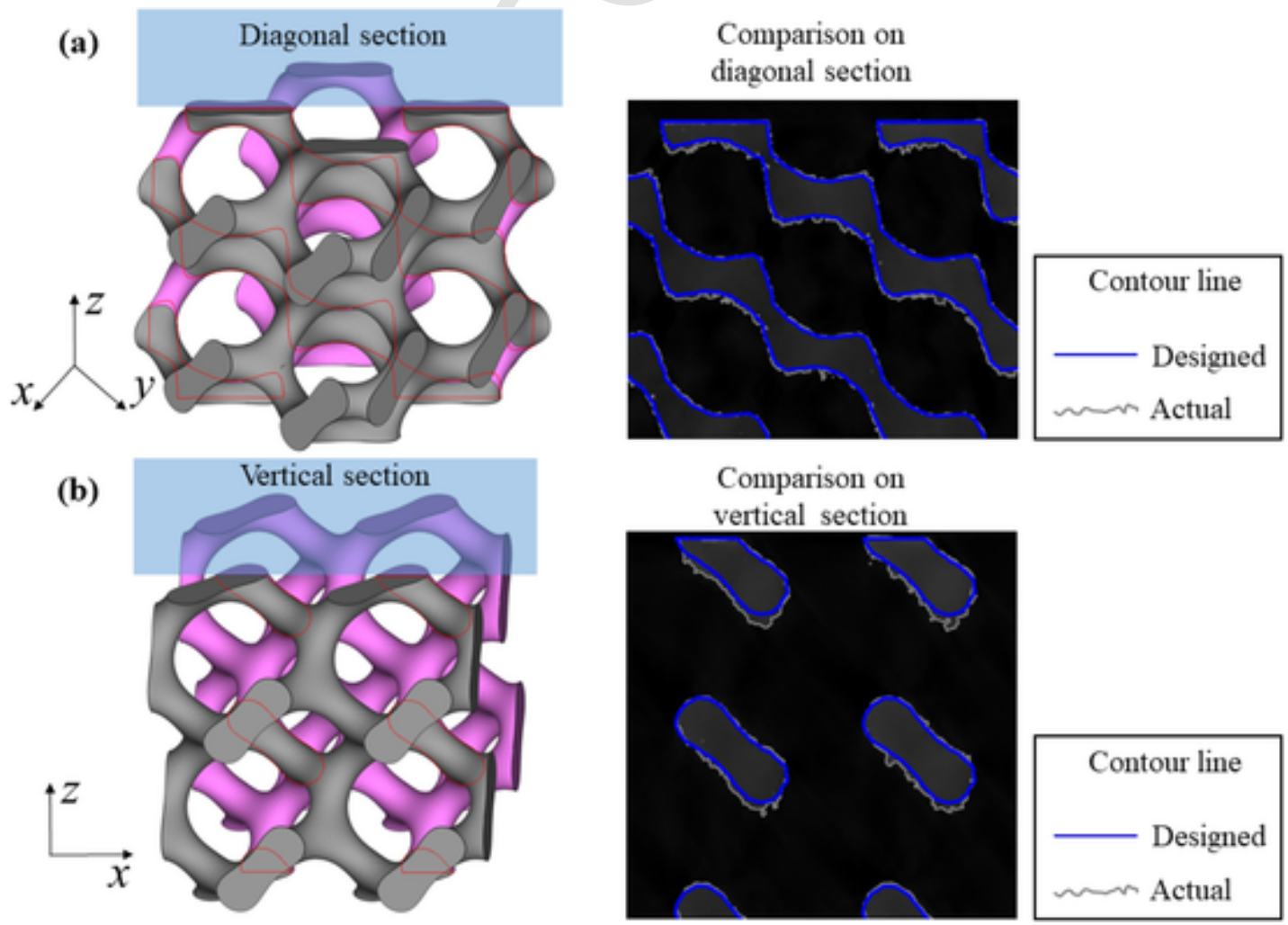

Fig. 4. The typical contour comparison between nominal and actual GCS models on different sections: (a) diagonal section and (b) vertical section. 
(a)

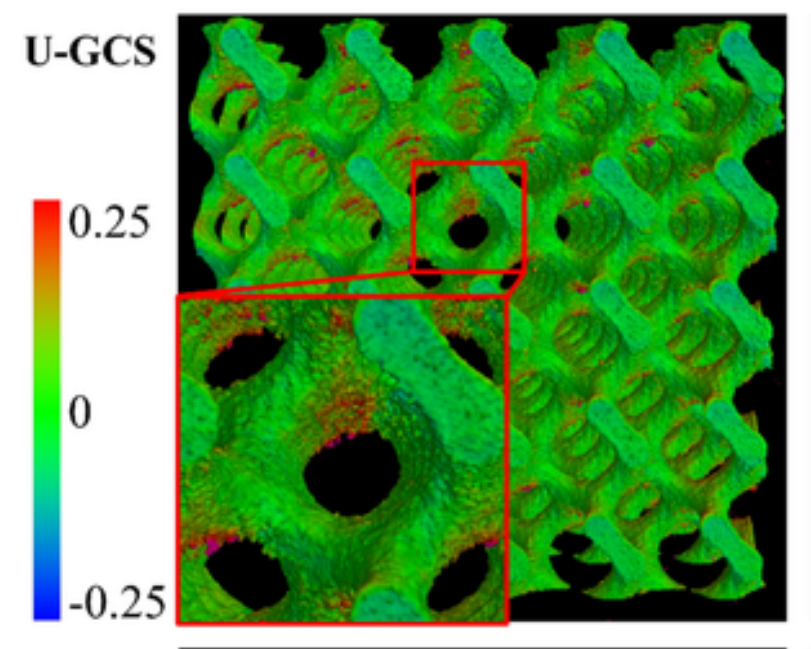

(b)
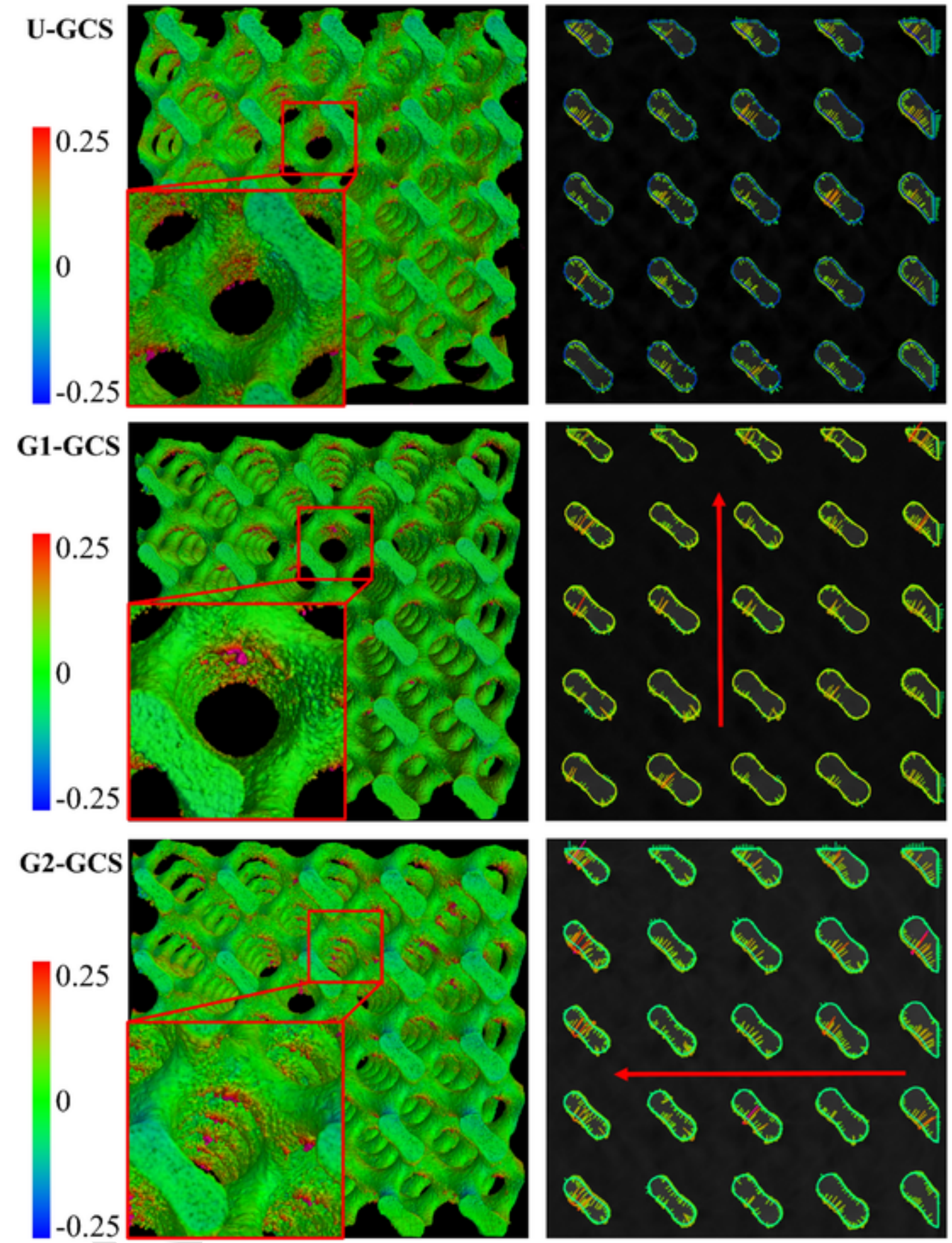

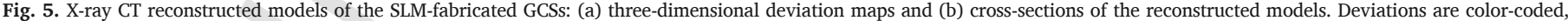
while the arrows in (b) denote the gradient direction.

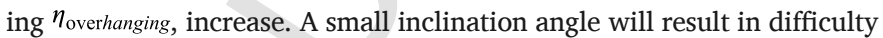
of manufacturing and even collapse of the as-built layer, due to a lack of support and the larger molten pool, as reported by Mazur et al., 2016b. Han et al. (2018) also presents a study of AlSi10Mg circular structures fabricated by SLM and the surface roughness was found to increase with the decrease in the face angle in the sequence of the staircase effect and bonded powder particles, associated with the for- mation of dross defects. In this study, the upper inner wall of sphere-like pores is characterized by low inclination angles. Therefore, the consequences associated with overhangs (i.e. exceeded downward profile and increased surface roughness) are indeed present.

Furthermore, Eq. (5) implies that the overhanging rate also depends on the strut diameter. When 3D printing a solid part, the effect of inclined surfaces is limited to the surface roughness; the effect of inclined 


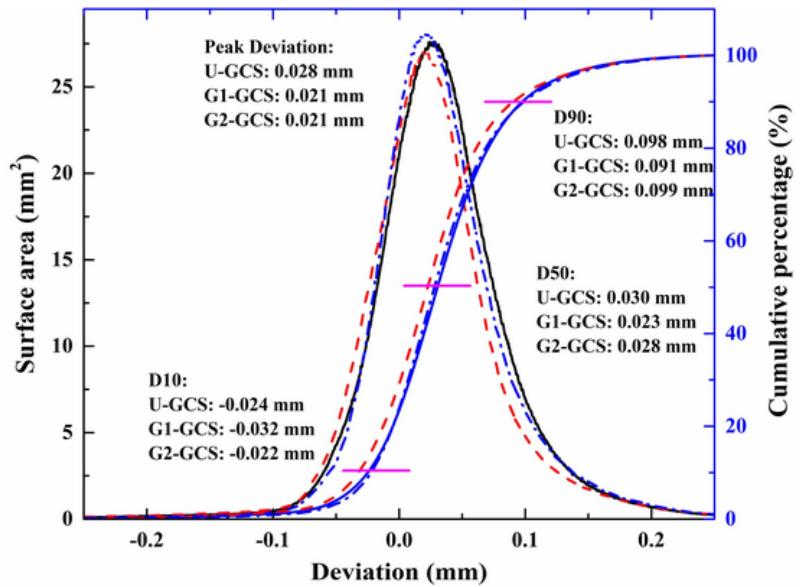

Fig. 6. Distribution of surface deviations of uniform and graded GCSs.

surfaces on the actual weight or volume of the solid part is not significant. For the cellular structures, particularly those with a low RD, strut diameter is comparable to the overhanging length; the overhanging rate and its effect on manufacturing fidelity are therefore much higher in cellular structures. Besides, Al-Ketan et al. (2018) compared the deviations between as-built samples and the CAD designed TPMS cellular structures, and noted that the deviation is stringently related to the surface area of each TPMS structure. The small strut diameter for the same unit cell size indicates lower $\mathrm{RD}$ and higher specific surface area as shown in Fig. 8, thus increasing the interaction between solidified structure and the loose powder particles and the occurrence of bonded powder particles.

Moreover, the gradient magnitude in cellular structures will also affect the degree of overhanging. As shown in Fig. 7(c), for a graded strut with a diameter gradient $\Delta t$, it is inevitable to have a larger di- ameter below and smaller diameter above for achieving an equivalent $\mathrm{RD}$. The length of the overhanging area becomes $l-\Delta t / 21-\Delta \mathrm{t} / 2$, which is lower than the overhang length in the uniform case. Therefore, higher gradients result in smaller overhangs, which reduces the occurrence of bonded loose powders. The density of G2-GCS is constant along the building direction, and thus, deviations are the same level as U-GCS. The gradient along building direction in G1-GCS provides better support for the new layer and therefore shortens the overhangs, resulting in better control of the outline and a lower deviation between the as-built and designed CAD models.

\subsection{Further demonstration}

To validate the analysis the effect of gradients on manufacturing fidelity given by Eqs. (4) and (5), the X-ray CT reconstructed models were segmented by unit cell layer along the building and gradient direction, respectively. Layer numbering is defined along the building direction and the decreasing density direction. For U-GCS and G1-GCS, these two directions are the same, and thus one set of data is sufficient. However, two separate sets of data are required for G2-GCS, as the two directions (building and gradient) are perpendicular to each other. Fig. 9(a) shows the schematic of G2-GCS segmented into layers; subscripts $b$ and $g$ indicate the building and gradient directions, respectively. For each G2-GCS (b) layer along the building direction, RD is constant, while RD of G2-GCS ( $\mathrm{g}$ ) along the gradient direction is gradually changing. For each layer, the CT measured volume is compared to the volume specified in the engineering design. The percentage deviation of the volume was calculated by dividing the deviation by the specified volume; percentage deviation is presented in Fig. 9(b).

For both U-GCS and G2-GCS (b), the percentage deviation of VF ranges from $11.00 \%$ to $12.27 \%$ for all layers. Volume deviation of G2-GCS shows no obvious difference with U-GCS, which confirms the previous finding that the gradient VF perpendicular to the building direc-

(a)

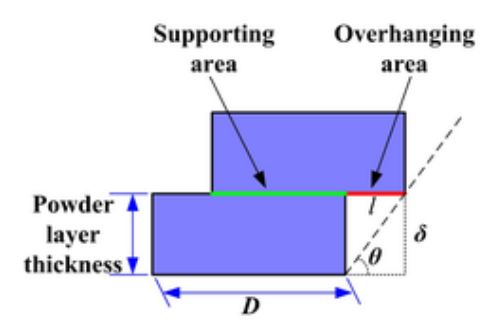

(c) Uniform strut

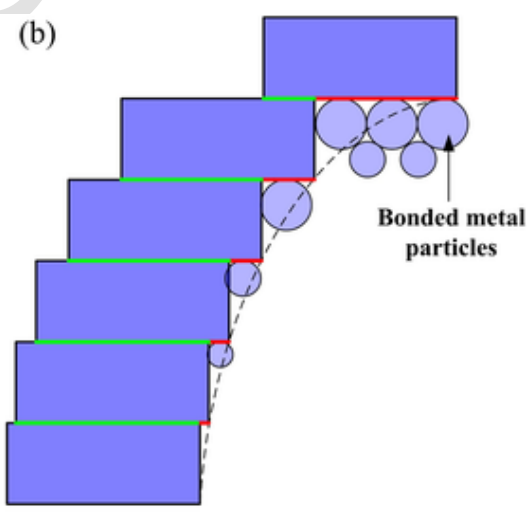

Graded strut
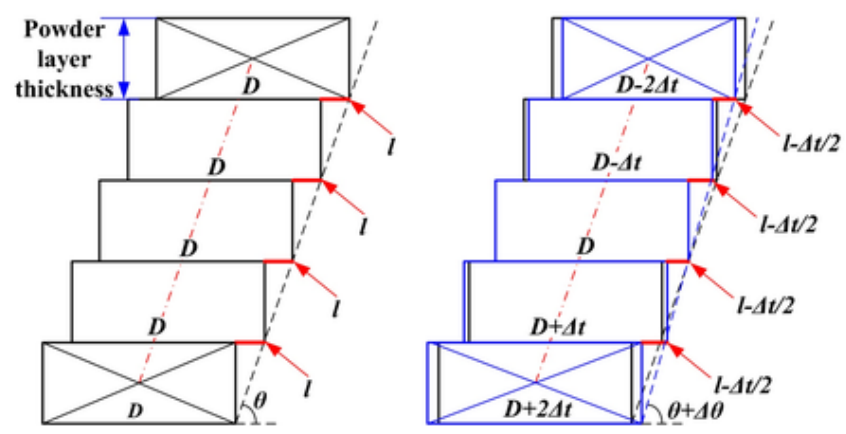

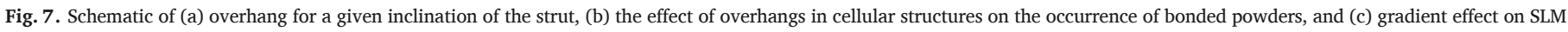
manufacturing fidelity of cellular structures. 


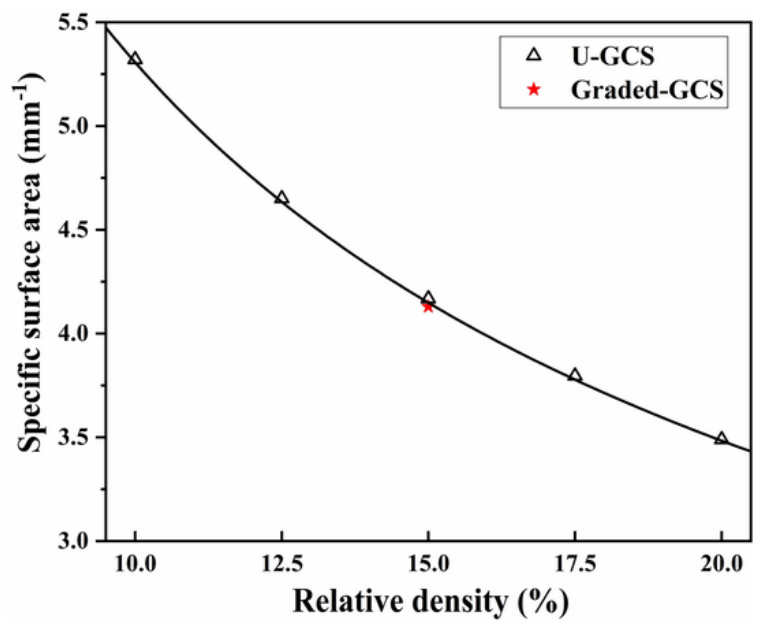

Fig. 8. The specific surface area per volume for the U-GCSs with different RD and Graded-GCSs (G1-GCS and G2-GCS).
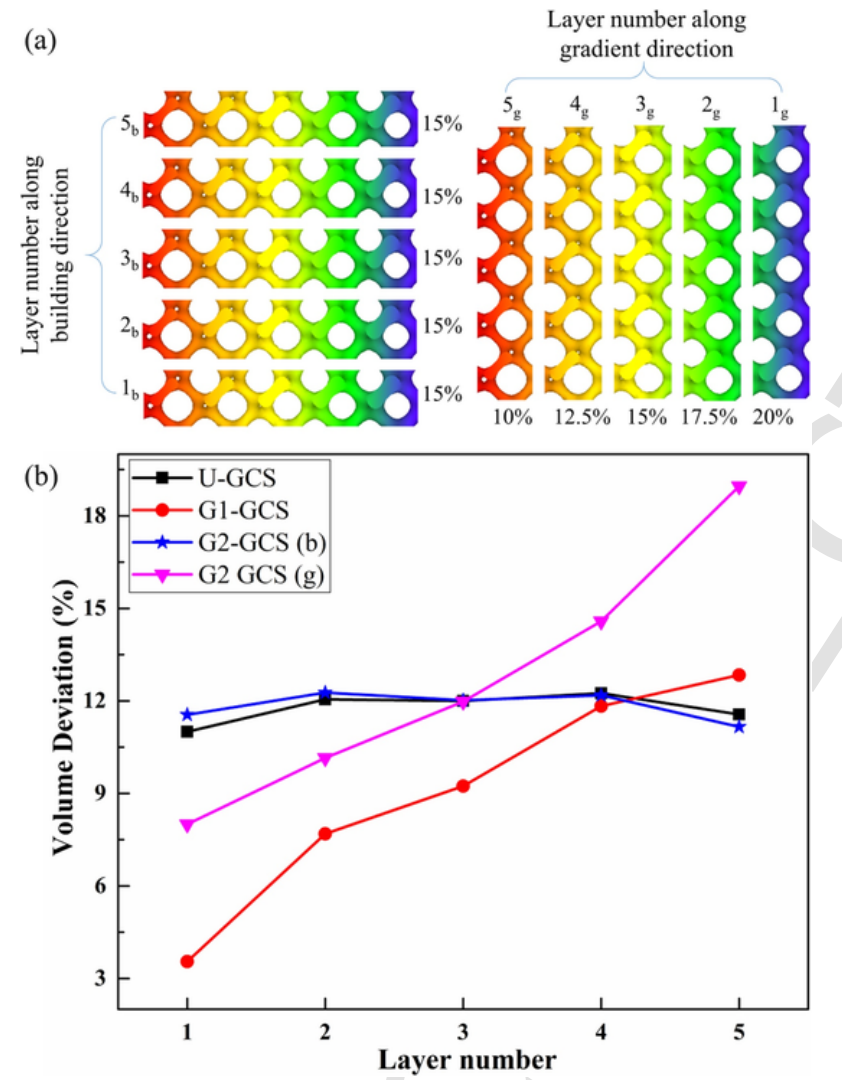

Fig. 9. Volume deviation between CT-reconstructed and engineering design: (a) schematic of G2-GCS segmented into layers and (b) percentage deviation of each layer in GCSs.

tion does not affect the actual volume of as-built GCSs, due to the same designed VF for each layer.

For the layers of G1-GCS and G2-GCS (g), volume deviations significantly increase from $3.55 \%$ to $12.84 \%$ and from $8.00 \%$ to $18.96 \%$, respectively, as the VF of each layer decreases. This observation confirms the findings in Section 3.3 and analysis illustrated in Fig. 7. Furthermore, the SLM technique and the surface area of cellular structures can also explain these findings. For the layers with low RD, high laser energy is concentrated in a smaller strut, which leads to a large melt pool and increases the diameter of as-built struts and the occurrence of adhered powders, as reported by Hussein et al. (2013). Moreover, the surface area per volume of cellular structures with low RD is greater than the surface area per volume of high RD structures, resulting in more bonded powders. It is worth noting that the volume deviation of all layers in G1-GCS is noticeably lower than the layers of G2-GCS with the same $\mathrm{RD}$, which can be attributed to the density gradient. As discussed in Section 3.3, the decreasing density alleviates the extent of overhanging; thus, for the layers in G1-GCS, the decreasing density along the building direction will effectively improve the manufacturing fidelity. From the analysis and discussion above, it is also predictable that increasing the density along the building direction will increase the overhanging area, resulting in more bonded powers and lower manufacturing fidelity.

\subsection{Assessment of manufacturing fidelity}

Regardless of the uneven porosity distribution of the AM samples, the values of RD and VF should be approximately the same. Based on the discussion above, the manufacturing fidelity of GCSs can be reflected by the deviation of $\mathrm{RD}$ and VF to a certain extent: the higher the deviations, the worse the manufacturing fidelity. The deviation values are in direct proportion to specific surface area $\boldsymbol{S} / \boldsymbol{V}$ and the extent of overhanging as follows,

$\eta_{R D} \approx \eta_{V F} \propto \frac{S}{V} \cdot \eta_{\text {overhanging }}$

Specific surface area is related to the RD and topology of the unit cell. The GCS with higher RD has proven to have a lower specific surface area, as shown in Fig. 8, resulting in higher manufacturing fidelity. In terms of topology, Al-Ketan et al. (2018) pointed out the sheet-TPMS based samples own higher specific surface area compared to the skeletal-TPMS based structures, leading to more severe deviations from the CAD design.

Due to the lack of support and insufficient cooling, local distortion is likely to happen at the overhanging position. Mazur et al., 2016 observed local collapse in unsupported areas of cantilever strut elements during the AM process. For porous/lattice structures, as the struts are supported at both ends, horizontal struts can be manufactured if the length is small enough, which is experimentally verified by Hernández-Nava et al. (2016). Nevertheless, the distortion and severe bonded powder will result in a higher deviation from the CAD design. The extent of overhanging mainly depends on the topology of the unit cell. Yan et al. (2014) noted that the circular struts of TPMS cellular structures can support themselves although there is still an extent of overhanging. However, without the horizontal struts, cellular structures with arbitrary strut length can be additively manufactured. An RD gradient in GCSs does not reduce the specific surface area as shown in Fig. 8, but increases the inclination angle of struts and shortens the overhangs, thus lowering the extent of overhanging and improving the manufacturing fidelity.

\section{Conclusion}

In this paper, we highlight two significant findings:

a)Three-dimensional deviation maps between the CT data and engineering designs show that bonded powder particles and considerable deviation occur at the upper inner wall of sphere-like pores with low inclination angles, due to the lack of support and insufficient cooling. The occurrence of bonded powders varies with geometry and RD. It is shown that a negative density gradient along the building direction can reduce the amount of bonded powder particles.

b)A mathematical model was established to analyse the effect of geometry parameters of the struts on manufacturing fidelity. It is demonstrated that manufacturing fidelity of SLM-built U-GCSs and G-GCSs depends on the geometry, including the inclination angle of struts, the relative density, and the gradient direction. 
The developed mathematical model provides an efficient way to assess the manufacturing fidelity of porous/lattice/cellular structures before the SLM process. The model can be applied to other powder bed AM technologies (for example, electronic beam melting). These findings also provide a reference for the design and optimization of cellular structures: for the cellular structures with fixed relative density, overhangs should be avoided and a gradient with decreasing density along building direction improves manufacturing fidelity.

\section{Acknowledgements}

The study was supported by the National Natural Science Foundation of China (Grant number 51671091), the independent R\&D subjects of Huazhong University of Science and Technology (Grant number 2017JYCXJJ005), and the MSCA Individual Fellowship FlexCT project (Grant number 752672).

\section{References}

Al-Ketan, O., Rowshan, R., Abu Al-Rub, R.K., 2018. Topology-mechanical property relationship of 3D printed strut, skeletal, and sheet based periodic metallic cellular materials. Addit. Manuf. 19, 167-183.

Al-Saedi, D.S.J., Masood, S.H., Faizan-Ur-Rab, M., Alomarah, A., Ponnusamy, P., 2018. Mechanical properties and energy absorption capability of functionally graded F2BCC lattice fabricated by SLM. Mater. Des. 144, 32-44.

Concli, F., Gilioli, A., 2019. Numerical and experimental assessment of the mechanical properties of 3D printed 18-Ni300 steel trabecular structures produced by Selective Laser Melting - a lean design approach. Virtual Phys. Prototyp. 14, 267-276.

Han, Q., Gu, H., Soe, S., Setchi, R., Lacan, F., Hill, J., 2018. Manufacturability of AlSi10Mg overhang structures fabricated by laser powder bed fusion. Mater. Des. 160, 1080-1095.

Hao, L., Raymont, D., Yan, C., Hussein, A., Young, P., 2011. Design and additive manufacturing of cellular lattice structures. In: The International Conference on Advanced Research in Virtual and Rapid Prototyping (VRAP), pp. 249-254.

Hernández-Nava, E., Smith, C.J., Derguti, F., Tammas-Williams, S., Leonard, F., Withers, P.J., Todd, I., Goodall, R., 2016. The effect of defects on the mechanical response of Ti-6Al-4V cubic lattice structures fabricated by electron beam melting. Acta Mater. 108, 279-292.

Hussein, A., Hao, L., Yan, C., Everson, R., Young, P., 2013. Advanced lattice support structures for metal additive manufacturing. J. Mater. Process. Technol. 213, 1019-1026.

Kapfer, S.C., Hyde, S.T., Mecke, K., Arns, C.H., Schroder-Turk, G.E., 2011. Minimal surface scaffold designs for tissue engineering. Biomaterials 32, 6875-6882.
Kruth, J.-P., Mercelis, P., Van Vaerenbergh, J., Craeghs, T., 2007. Feedback control of selective laser melting. In: Proceedings of the 3rd International Conference on Advanced Research in Virtual and Rapid Prototyping, pp. 521-527.

Kruth, J., Vandenbroucke, B., Van Vaerenbergh, J., Mercelis, P., 2005. Benchmarking of different SLS/SLM processes as rapid manufacturing techniques. Laser $13 \mathrm{D}$.

Luzzati, V., Spegt, P., 1967. Polymorphism of lipids. Nature 215, 701-704.

Li, S., Zhao, S., Hou, W., Teng, C., Hao, Y., Li, Y., Yang, R., Misra, R.D.K., 2016. Functionally graded Ti-6Al-4V meshes with high strength and energy absorption. Adv. Eng. Mater. 18, 34-38.

Mancisidor, A.M., Garciandia, F., Sebastian, M.S., Álvarez, P., Díaz, J., Unanue, I., 2016. Reduction of the residual porosity in parts manufactured by selective laser melting using skywriting and high focus offset strategies. Phys. Procedia 83, 864-873.

Maskery, I., Aremu, A., Parry, L., Wildman, R., Tuck, C., Ashcroft, I., 2018. Effective design and simulation of surface-based lattice structures featuring volume fraction and cell type grading. Mater. Des. 155, 220-232.

Mazur, M., Leary, M., McMillan, M., Elambasseril, J., Brandt, M., 2016a. SLM additive manufacture of $\mathrm{H} 13$ tool steel with conformal cooling and structural lattices. Rapid Prototyp. J. 22, 504-518.

Mazur, M., Leary, M., Sun, S., Vcelka, M., Shidid, D., Brandt, M., 2016b. Deformation and failure behaviour of Ti-6Al-4V lattice structures manufactured by selective laser melting (SLM). Int. J. Adv. Manuf. Technol. 84, 1391-1411.

Montazerian, H., Davoodi, E., Asadi-Eydivand, M., Kadkhodapour, J., Solati-Hashjin, M., 2017. Porous scaffold internal architecture design based on minimal surfaces: a compromise between permeability and elastic properties. Mater. Des. 126, 98-114.

Sarker, A., Tran, N., Rifai, A., Elambasseril, J., Brandt, M., Williams, R., Leary, M., Fox, K., 2018. Angle defines attachment: switching the biological response to titanium interfaces by modifying the inclination angle during selective laser melting. Mater. Des. 154, 326-339.

Scherer, M.R.J., 2013. Double-Gyroid-Structured Functional Materials: Synthesis and Applications, Springer Science \& Business Media, Switerland.

Sing, S.L., Wiria, F.E., Yeong, W.Y., 2018. Selective laser melting of lattice structures: a statistical approach to manufacturability and mechanical behavior. Robot Comput. Integr. Manuf. 49, 170-180.

Strano, G., Hao, L., Everson, R.M., Evans, K.E., 2013. Surface roughness analysis, modelling and prediction in selective laser melting. J. Mater. Process. Technol. 213, 589-597.

Wang, D., Yang, Y., Liu, R., Xiao, D., Sun, J., 2013. Study on the designing rules and processability of porous structure based on selective laser melting (SLM). J. Mater. Process. Technol. 213, 1734-1742.

Yan, C., Hao, L., Hussein, A., Raymont, D., 2012. Evaluations of cellular lattice structures manufactured using selective laser melting. Int. J. Mach. Tools Manuf. 62, 32-38.

Yan, C.Z., Hao, L., Hussein, A., Young, P., Raymont, D., 2014. Advanced lightweight 316L stainless steel cellular lattice structures fabricated via selective laser melting. Mater. Des. 55, 533-541.

Yánez, A., Cuadrado, A., Martel, O., Afonso, H., Monopoli, D., 2018. Gyroid porous titanium structures: a versatile solution to be used as scaffolds in bone defect reconstruction. Mater. Des. 140, 21-29.

Yang, L., Mertens, R., Ferrucci, M., Yan, C., Shi, Y., Yang, S., 2019. Continuous graded Gyroid cellular structures fabricated by selective laser melting: design, manufacturing and mechanical properties. Mater. Des. 162, 394-404.

Yang, L., Yan, C., Han, C., Chen, P., Yang, S., Shi, Y., 2018. Mechanical response of a triply periodic minimal surface cellular structures manufactured by selective laser melting. Int. J. Mech. Sci. 148, 149-157. 